\title{
Design of fusion protein for efficient preparation of cyanovirin-n and rapid enrichment of pseudorabies virus
}

\author{
Bin Wang $\cdot$ Zhi Yang $\cdot$ Dan Gao $\cdot$ Fei Wang $\cdot$ Min Liu $\cdot$ Guanjun Chen • \\ Lixin Ma $\cdot$ Xiaolan Yu
}

Received: 3 January 2021 / Accepted: 23 April 2021 / Published online: 9 May 2021

(C) The Author(s), under exclusive licence to Springer Nature B.V. 2021

\begin{abstract}
Objective Cyanovirin- $\mathrm{N}(\mathrm{CVN})$ is a cyanobacterial protein with potent neutralizing activity against enveloped virus. To achieve the economic and functional production of $\mathrm{CVN}$, the $\mathrm{CVN} \mathrm{N}$-terminally fused with CL7(A mutant of the Colicin E7 Dnase) was utilized to improve the solubility and stability of CVN fusion protein (CL7-CVN). Additionally, to improve the detection limit of existing PRV diagnostic
\end{abstract}

Bin Wang, Zhi Yang and Dan Gao have contributed equally to this work.

Supplementary Information The online version contains supplementary material available at https://doi.org/10.1007/ s10529-021-03141-x.

B. Wang - Z. Yang $\cdot$ D. Gao $\cdot$ F. Wang .

M. Liu · G. Chen · L. Ma $(\bowtie) \cdot$ X. Yu $(\varangle)$

State Key Laboratory of Biocatalysis and Enzyme

Engineering, Hubei University, Wuhan 430062, China

e-mail: malixing@hubu.edu.cn

$\mathrm{X} . \mathrm{Yu}$

e-mail: yxiaolan@163.com

B. Wang

e-mail: 15107151836@163.com

Z. Yang

e-mail: 18371269740@163.com

D. Gao

e-mail: gaodan1208@163.com assays, CL7-CVN was used for Pseudorabies virus (PRV) enrichment from larger sample volumes.

Results CVN fused with CL7 was efficiently expressed at a level of $\sim 40 \%$ of the total soluble protein in $E$. coli by optimizing the induction conditions. Also, the stability of CVN fusion protein was enhanced, and $10 \mathrm{mg}$ of CVN with a purity of 99\% were obtained from $1 \mathrm{~g}$ of cells by one-step affinity purification with the digestion of HRV 3C protease. Moreover, both purified CVN and CL7-CVN could effectively inhibit the infection of PRV to PK15 cells. Considering the bioactivity of CL7-CVN, we explored a strategy for PRV enrichment from larger samples. Conclusions CL7 effectively promoted the soluble expression of CVN fusion protein and improved its stability, which was meaningful for its purification and
F. Wang
e-mail: fhda142@163.com
M. Liu
e-mail: liuminm8@163.com
G. Chen
e-mail: 1653630108@qq.com 
application. The design of CVN fusion protein provides an efficient approach for the economical and functional production of CVN and a new strategy for PRV enrichment.

Keywords CVN · CL7 - Soluble expression · Rapid purification · Anti-PRV · Virus enrichment

\section{Introduction}

Globally, millions of human beings and animals are exposed to the enveloped viruses, threatening human health and the economy. Currently, effective antiviral drugs for the treatment of enveloped viruses are unavailable. Vaccines are used to inhibit the development of the epidemics, but the vaccines cannot eliminate the virus infection when the mutant strains appear frequently. Cyanovirin-n (CVN) initially discovered and isolated from cyanobacteria shows to be an effective antiviral protein for multitudinous enveloped viruses, such as Influenza A and B viruses, Herpes simplex virus type-1, and Human immunodeficiency virus (Smee et al. 2008; Buffa et al. 2009; Tiwari et al. 2009). CVN combines the highly glycosylated proteins of the enveloped virus with high affinity via two carbohydrate-binding domains, which prevents the virus from invading and adhering (Lusvarghi et al. 2016). Importantly, the antiviral activity of CVN is unaffected by the virus mutations that escape from immune system suppression. Therefore, its effective bioactivity and stable physicochemical properties make it possible for CVN to be a potential antiviral drug. Currently, CVN has been tried to be produced by eukaryotic expression system and prokaryotic expression system (Mori et al. 2002; Sexton et al. 2006). However, there are still some obstacles to the efficient preparation of CVN due to the formation of dimers, glycosylation modification, and downstream processing (Gao et al. 2010; Madeira et al. 2016). Even so, E. coli is still the preferred system for heterologous proteins production because of its simple culture conditions, no special instruments, convenient operation and short production cycle (Wurm et al. 2017; Kleiner-Grote et al. 2018).

The CL7/Im7 purification system is used for onestep purification of various biological molecules (Vassylyeva et al. 2017). When we purified the proteins with the purification system, we inadvertently found that CL7 promoted the expression of some specific proteins, and could even resist $\sim 90{ }^{\circ} \mathrm{C}$. Besides, CVN can remain in boiling water for $15 \mathrm{~min}$. Here, CVN fused with CL7 is utilized to improve the soluble expression and purification of CVN (Vassylyeva et al. 2017). The results suggested CL7-CVN was highly and stably expressed with excellent stability in E. coli. Moreover, the high purity and activity CVN could be obtained by one-step affinity purification with Ni-NTA column. Furthermore, considering the biology of CVN fusion protein and the high affinity of CL7/Im7 (Kd $\left.\sim 10^{-14}-10^{-17} \mathrm{M}\right)$ (Vassylyeva et al. 2017), we proposed a strategy for PRV enrichment based on the affinity of CL7-CVN to enveloped viruses and $\operatorname{Im} 7$ Beads to improve the virus detection rate.

\section{Materials and methods}

Strains, viruses and cells

The E. coli strains DH5 $\alpha$ and Rosetta (DE3) were purchased from Sangon (Shanghai, China) and preserved by our laboratory. PRV (The UL21 gene of PRV was replaced by EGFP, $\triangle$ UL21/EGFP) and PK15 cells (Porcine kidney 15 cell line) were kindly provided by Huazhong Agricultural University, Wuhan, Hubei, China.

Protein expression and purification

According to the codon usage bias of E. coli, the nucleotide sequence of CVN (GenBank: KJ632500) was optimized and synthesized for protein expression in E. coli. The designed His-CVN and CL7-CVN were cloned into the pET28a plasmid. Then, the Rosetta(DE3) strains harboring these plasmids were grown at $37^{\circ} \mathrm{C}$ in LB medium with $50 \mu \mathrm{g} \cdot \mathrm{ml}-1$ Kanamycin until the culture reached an OD600 $=0.6$, then induced with $1 \mathrm{mM}$ IPTG at $37^{\circ} \mathrm{C}$ for $4 \mathrm{~h}$. The cells were harvested and disrupted for testing protein expression by Tricine-SDS-PAGE (Fig. 1b).

To further improve the solubility of CVN fusion protein, the expression conditions for CVN fusion protein in E. coli Rosetta (DE3) were optimized by testing the supernatant and precipitates of the cells induced at various culture temperatures $(18,28,37$ 
${ }^{\circ} \mathrm{C}$ ), induction periods (12, 16 and $20 \mathrm{~h}$ ), and IPTG concentrations $(0.25,0.5,1.0 \mathrm{mM})$.

The typical procedures for the purifications of CVN and CL7-CVN were illustrated in (Fig. 2a). The supernatant of cell lysates CL7-CVN was treated at 80 ${ }^{\circ} \mathrm{C}$ for $30 \mathrm{~min}$. Then, the purer supernatant of CL7CVN was separated by centrifugation and loaded onto Ni-NTA column (GE Healthcare) for purification. The nonspecifically bound proteins were eluted with the buffer (10-30 mM imidazole, $20 \mathrm{mM}$ Tris- $\mathrm{HCl}$, $500 \mathrm{mM} \mathrm{NaCl}, \mathrm{pH}$ 8.0) until the OD280 reached a base line. Subsequently, CL7-CVN was eluted with the buffer (300 mM imidazole, $20 \mathrm{mM}$ Tris-HCl, $500 \mathrm{mM}$ $\mathrm{NaCl}, \mathrm{pH}$ 8.0). Briefly, CVN was released from the column with the digestion of HRV 3C protease, and undigested CL7-CVN, CL7 and HRV 3C protease stayed on the column.

\section{Real-time PCR assay}

Equal mole of purified proteins $(0.25 \mu \mathrm{M}$ of CL7, CVN, and CL7-CVN) were added to $100 \mu \mathrm{L}$ of 100 TCID50/mL PRV and incubated at $37{ }^{\circ} \mathrm{C}$ for $1 \mathrm{~h}$. Then, PK15 cells were incubated with PRV for $2 \mathrm{~h}$. After changing the medium, culture was continued for $24 \mathrm{~h}$. Afterwards, all of the RNA were carefully extracted from PK15 cells. Ultimately, cDNA was rapidly synthesized for real-time PCR analysis. The
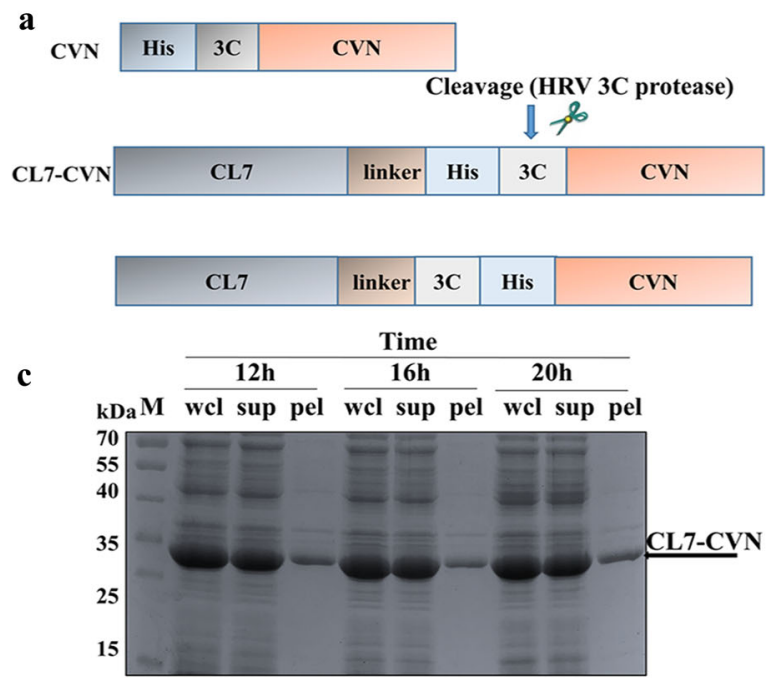

Fig. 1 The design and expression of CVN fusion protein. a Structure designs of CVN fusion protein for the expression of His-CVN and CL7-CVN. b The soluble expression of CL7CVN in E. coli. c Optimization of CL7-CVN expression assay was performed under the following conditions: 5-min activation of Taq DNA polymerase at $95{ }^{\circ} \mathrm{C}$, followed by 40 cycles of $15 \mathrm{~s}$ at $95^{\circ} \mathrm{C}, 45 \mathrm{~s}$ at $60{ }^{\circ} \mathrm{C}$. The standard curve assay for mRNA was also performed.

$50 \%$ tissue culture infective dose assays

The PK15 cells were placed in 96-well plates and incubated with $100 \mu \mathrm{L}$ PRV particles at $37^{\circ} \mathrm{C}$ for $1 \mathrm{~h}$. After removing the culture medium, different concentrations $(0.25,0.5,1,2 \mu \mathrm{M})$ of CL7-CVN and CVN were added to incubate with cells for further $48 \mathrm{~h}$. After the incubation period, the viruses were harvested and used for TCID50 assays.

\section{Enzyme-linked immunosorbent assay (ELISA)}

ELISA plates were evenly incubated with PRV particles in PBS at $37^{\circ} \mathrm{C}$ for $2 \mathrm{~h}$. The PBS containing $2 \%$ BSA was then employed to block at $37^{\circ} \mathrm{C}$ for $1 \mathrm{~h}$. Then, the different concentrations of CL7-CVN, CVN, and CL7 were added to ELISA plates and incubated at $37{ }^{\circ} \mathrm{C}$ for $1 \mathrm{~h}$. The HRP substrates and Anti-His-tag HRP antibody were used to detect the conjugated CL7-CVN, CVN and CL7. Finally, the OD450 values were measured by Genios (Fig. 3d).

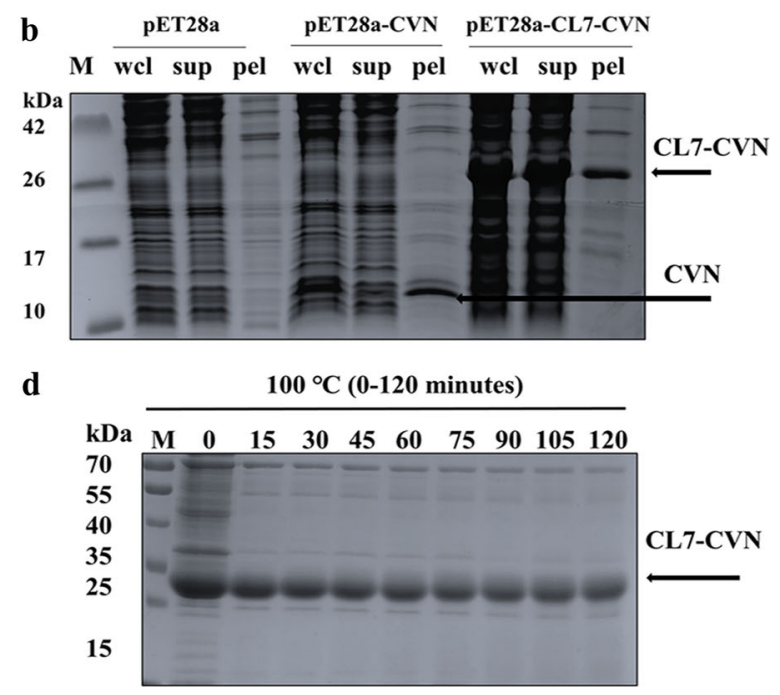

condition (Temperature, IPTG, Time).wcl, whole cell lysates; sup, supernatant of cell lysates; pel, pellet of cell lysates (pel). d Changes of supernatant protein in cell lysates treated at $100^{\circ} \mathrm{C}$ for $0-120 \mathrm{~min}$ 


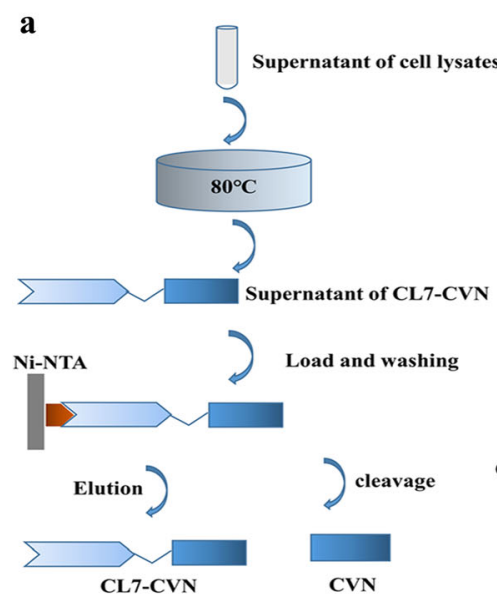

Fig. 2 Protein purification. a one-step affinity purification process for CVN and CL7-CVN. b, c One-step affinity purification of CL7-CVN and CVN. Sup, supernatant of cell lysates; H-Sup, supernatant of cell lysates heated; column, CL7CVN on column; Cleavage, cleavage of CL7-CVN on the

Enrichment and detection of PRV

The highly purified $\operatorname{Im} 7$ was concentrated and immobilized for protein immobilization onto the Sulfo-Link (iodo-acetyl activated) 6B agarose beads as described in the study (Vassylyeva et al. 2017). The typical concentration of the immobilized Im7-unit was $\sim 15$ $\mathrm{mg} / \mathrm{ml}$ beads $(\sim 0.6 \mathrm{mM})$. Then, $\operatorname{Im} 7$ Beads were used for the enrichment of PRV.

The enrichment strategy was conveniently performed to capture and identify PRV (Fig. 4a). Briefly, Im7 Beads and CL7-CVN were added to the samples containing PRV particles and then incubated $30 \mathrm{~min}$ through gentle shaking at room temperature. Afterwards, the generated complexes (Im7 Beads-CL7CVN-Virus) were rapidly separated by centrifugation. The viral DNA of the supernatant and pellet were extracted and measured by qPCR as described previously.

\section{Results}

\section{Expression of CVN and CL7-CVN}

After induction at $37^{\circ} \mathrm{C}$ for $4 \mathrm{~h}, \mathrm{CL} 7-\mathrm{CVN}$ was highly expressed in E. coli, and most of CL7-CVN were found in the lysate supernatant (Fig. 1b). In contrast, column; FT, flow through; EL, eluate; M, protein marker. d Western blot was used to detect the changes of CVN and CL7$\mathrm{CVN}$ in serum. $\mathbf{e}$ The relative levels represent quantification of d using Image $\mathbf{J}$ software

CVN without CL7 were basically in the lysate precipitation. The $N$-terminal CL7 performed as effective solubility-enhancing fusion tag and could be used for the soluble expression of CVN. To further improve the soluble expression of CVN, we preliminarily optimized the induction conditions of CL7CVN expression (Temperature, IPTG concentration, Time). Briefly, the expression level was the higher ( $\sim 40 \%$ ) when IPTG of $0.25 \mathrm{mM}$ was used to induce culture at $18{ }^{\circ} \mathrm{C}$ for $20 \mathrm{~h}$ (Fig. 1c).

\section{Purification of CVN and CL7-CVN}

The thermal stability of CL7-CVN was determined before protein purification. The most of proteins were denatured under heating at $100{ }^{\circ} \mathrm{C}$, while the CVN fusion protein was intact, indicating that CL7-CVN had excellent thermal stability and could even exist at $100{ }^{\circ} \mathrm{C}$ for more than $2 \mathrm{~h}$ (Fig. 1d). Thus, we simplified the steps of CVN purification as described previously (Fig. 2a). In particular, the purity of CL7-CVN reached $\sim 80 \%$ after the supernatant of cell lysis is heated. Besides, the purity of CL7 -CVN reached 99\% via additional one-step affinity purification with $\mathrm{Ni}-\mathrm{NTA}$ (Fig. 2b). Moreover, $10 \mathrm{mg}$ of CVN with a purity of up to $99 \%$ were obtained from $1 \mathrm{~g}$ of E. coli by one-step affinity purification with the digestion of HRV 3C protease (Fig. 2c). These demonstrated that 


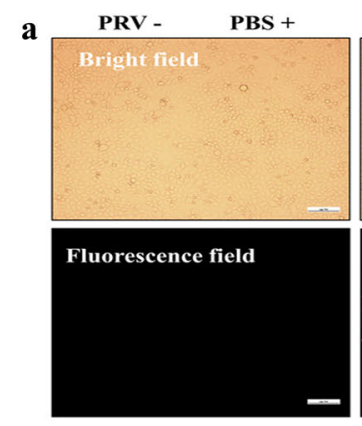

b

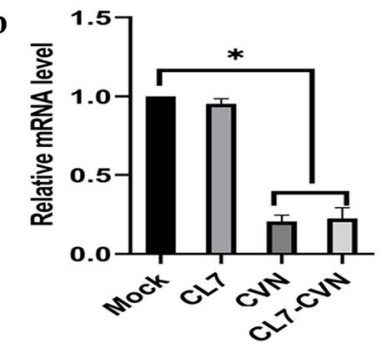

PRV + PBS +
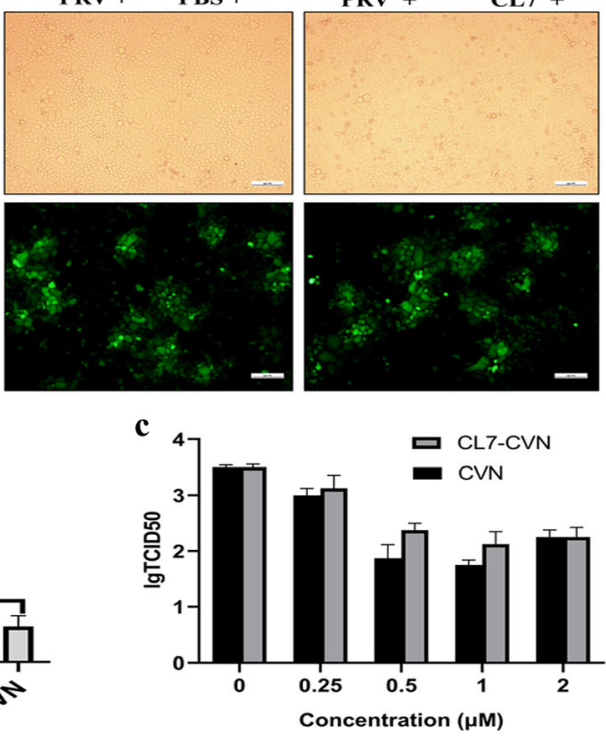
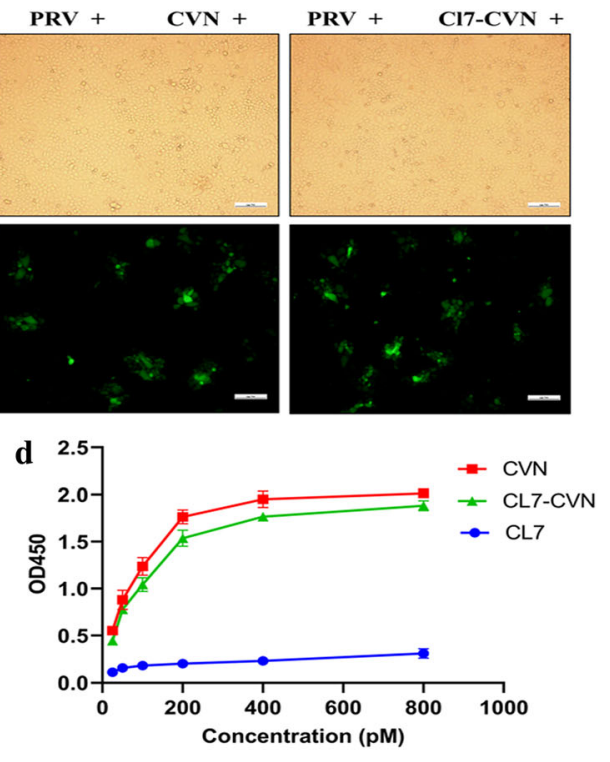

Fig. 3 Activity analysis of purified CVN and CL7-CVN. a Fluorescence microscopy images of PK15 cells infected by PRV treated with CL7-CVN, CVN, CL7 after culturing for $16 \mathrm{~h}$. b The relative levels of mRNA of PRV in the infected PK15 cells treated with CL7-CVN, CVN, CL7 $(* p<0.01)$. c TCID50 assay of antiviral activity of CVN or CL7-CVN. d ELISA was performed to analyze the affinity of CVN and CL7-CVN to PRV $\mathbf{a}$

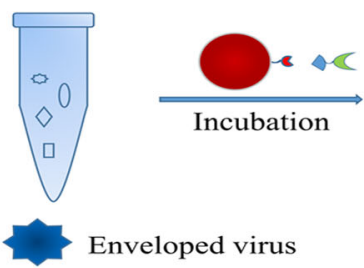

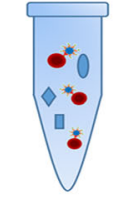

- $\operatorname{Im} 7$-Beads
Separation

Separation

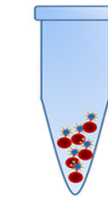

OC CL7-CVN

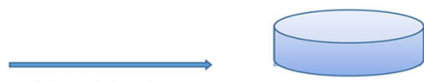

(Washing) qPCR Detection
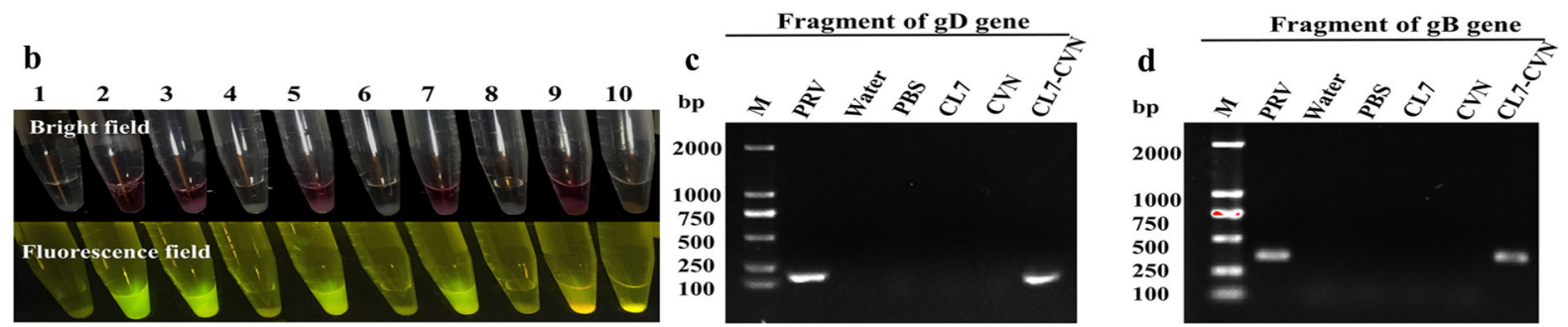

Fig. 4 The enrichment and detection of PRV. a Schematic of the strategy for the enrichment and detection of enveloped viruses using CL7-CVN with Im7 Beads. b The fluorescence intensity of PRV captured by $\operatorname{Im} 7$ Beads with PBS, CL7, CVN, and CL7-CVN. 1, Im7 Beads; 2, PRV sample; 3, PRV sample reacted with $\operatorname{Im} 7$ Beads and PBS; 4, PRV captured by $\operatorname{Im} 7$ Beads with PBS after washing; 5, PRV sample reacted with $\operatorname{Im} 7$ Beads and CL7; 6, PRV captured by Im7 Beads with CL7 after washing; 7, PRV sample reacted with $\operatorname{Im} 7$ Beads and CVN; 8, PRV captured by $\operatorname{Im} 7$ Beads with CVN after washing; 9, PRV sample reacted with $\operatorname{Im} 7$ Beads and CL7-CVN; 10, PRV captured by Im7 Beads with CL7-CVN after washing; c, d PCR analysis of PRV gene (206 bp fragment of gD gene, $331 \mathrm{bp}$ fragment of gB gene) captured by $\operatorname{Im} 7$ Beads with PBS, CL7, CVN, and CL7-CVN; PRV, positive control; Water, negative control 
CVN or CL7-CVN could be easily and economically purified using this system.

\section{Stability analysis of CVN fusion protein}

To further evaluate the stability of CL7-CVN, equal moles of CL7-CVN and CVN were respectively incubated with fresh chicken serum for $30 \mathrm{~h}$ at 37 ${ }^{\circ} \mathrm{C}$. Western blot results demonstrated that the protein level decreased with the increase of incubation time (Fig. 2d). Moreover, CVN decreased by half when it was incubated for $24 \mathrm{~h}$, yet CL7-CVN decreased by $\sim 28 \%$ when it was incubated for $30 \mathrm{~h}$ (Fig. 2e). CL7 significantly enhances the stability of CL7-CVN in serum, which is of great significance for its application.

Analysis of antiviral activity

After efficient production of proteins, the $\operatorname{PRV}(\Delta \mathrm{UL2} 2 / \mathrm{EGFP})$ was considered as a virus model to determine antiviral activity of CVN. PK15 cells were infected with PRV treated with CL7-CVN, CVN, and CL7 at $0.25 \mu \mathrm{M}$. Comparing fluorescence intensity of PK15 cells, we recognized a significant decrease in multiplication of PRV treated with CVN or CL7-CVN after $16 \mathrm{~h}$ of culture (Fig. 3a). Besides, we measured the mRNA of PRV gD in PK15 cells via qPCR assay. The relative virus mRNA levels of CVN and CL7-CVN groups decreased by $79.59 \%$ and 77.64\%, respectively, while CL7 did not significantly affect the mRNA level (Fig. 3b).

Besides, the TCID50 assay was used to assess the effect of CVN and CL7-CVN on PRV virulence. Compared to the negative control, PRV treated with $\mathrm{CVN}$ at $0.25,0.5,1$ and $2 \mu \mathrm{M}$ resulted in $0.5,1.63$, 1.75 and $1.25 \lg$ TCID50 reduction, respectively. Meanwhile, CL7-CVN also resulted in 0.375, 1.125, 1.375 and $1.25 \lg$ TCID50 reduction, respectively (Fig. 3c). The results showed that purified CL7-CVN and CVN inhibited PRV from infecting PK15 cells, indicating that the prepared CVN and CL7-CVN had eminent biological activity.

Furthermore, the ELISA was also implemented to characterize the affinity of CVN to PRV. CL7-CVN could bind to PRV as well as CVN, while CL7 could not recognize and combine PRV. Besides, with the decrease of CL7-CVN or CVN concentration, the
$\mathrm{OD}_{450}$ value decreased, suggesting its binding activity was dose-dependent (Fig. 3d).

Enrichment and detection of PRV

To improve the detection limit of existing PRV diagnostic assays, we explored a strategy for PRV enrichment utilizing $\operatorname{Im} 7$ beads with CL7-CVN. The process of virus isolation and enrichment was carried out as illustrated in (Fig. 4a). Im7 Beads treated with PRV sample and CL7-CVN had apparent fluorescence signal after washing. Conversely, the fluorescence signals of the control groups PBS, CL7, and CVN, weren't detected (Fig. 4b). Moreover, standard PCR assays for the enriched PRV were carried out. $206 \mathrm{bp}$ fragments of the $\mathrm{gD}$ gene, $331 \mathrm{bp}$ fragments of the $\mathrm{gB}$ gene, were respectively amplified from the PRV captured by $\operatorname{Im} 7$ Beads with CL7-CVN, whereas no fragments were observed when PRV samples were treated with $\operatorname{Im} 7$ Beads and PBS, CL7, CVN (Fig. 4c, d).

To further validate the reliability of our enrichment method, the amount of captured PRV was determined by qPCR. The PRV enrichment efficiency was calculated as the captured viral load divided by the initial viral load before the enrichment. A series of $100 \mu \mathrm{L}$ PRV samples $\left(10^{6}\right.$ viruses $\left./ \mu \mathrm{L}\right)$ was prepared for virus enrichment utilizing CL7, CVN, and CL7-CVN at $0.25 \mu \mathrm{M}, \operatorname{Im} 7$ Beads at $0.5 \mathrm{mg} / \mathrm{mL}$. The enrichment results showed that $\sim 90.78 \%$ of PRV were captured by $\operatorname{Im} 7$ Beads with CL7-CVN. Simultaneously, $20.93 \%$ of PRV were enriched by nonspecific physical adsorption by $\operatorname{Im} 7$ Beads with PBS (Fig. 5a). The efficiency of virus enrichment was calculated using different concentrations of CL7-CVN with $\operatorname{Im} 7$ Beads at $0.5 \mathrm{mg} / \mathrm{mL}$. The results showed that the enrichment efficiency increased with the increase of CVN concentration in a certain range, and $97.32 \%$ of virus was captured at $0.5 \mu \mathrm{M}$ of CL7-CVN (Fig. 5b).

\section{Capability of PRV enrichment}

To further assess the capability of our enrichment strategy, we tested the effect of sample size on the efficiency of virus enrichment. The $100 \mu \mathrm{L}$ PRV samples $\left(10^{6}\right.$ viruses $\left./ \mu \mathrm{L}\right)$ were respectively diluted to different final volumes for PRV enrichment. When the viral sample was diluted to $1000 \mu \mathrm{L}$, the enrichment efficiency of virus had little change and reached 


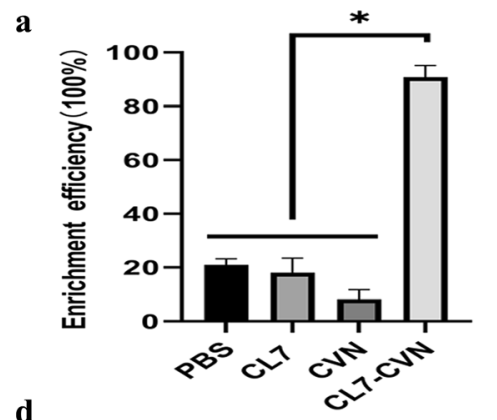

d

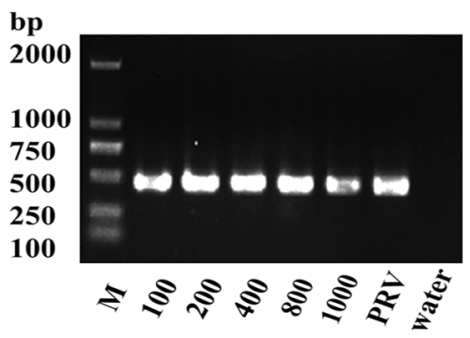

b

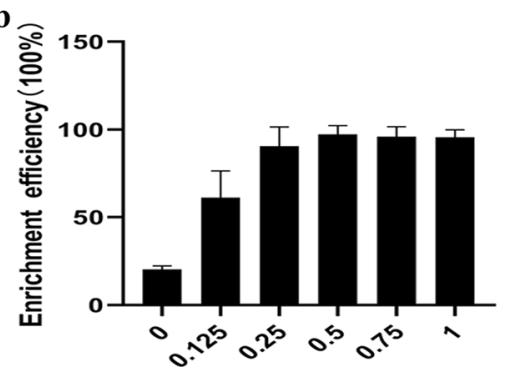

e

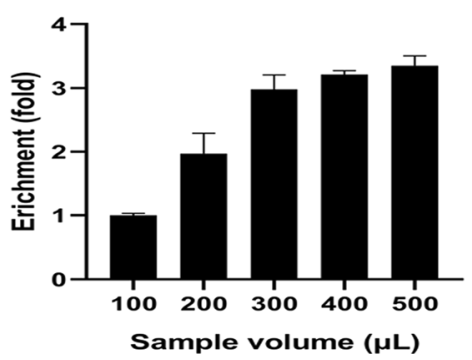

c

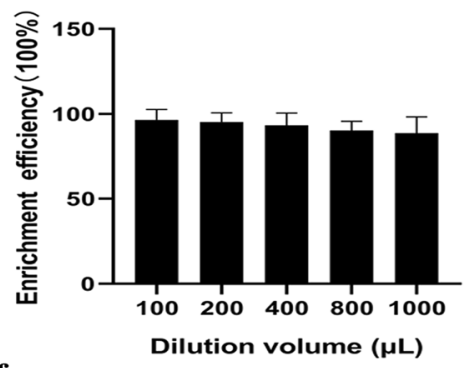

f

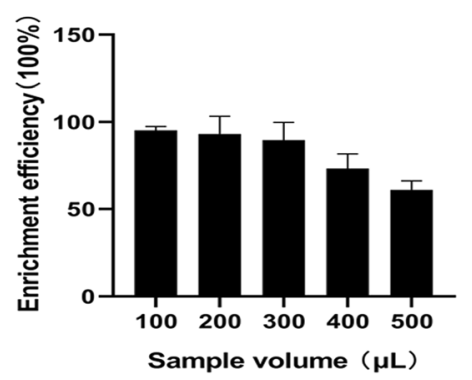

Fig. 5 Analysis of the enrichment capacity of PRV. a Different proteins were used to enrich virus samples $(* p<0.01)$. b Effect of CL7-CVN concentration on the enrichment efficiency. c Effect of sample size on the enrichment efficiency. d PCR

$88.72 \%$ (Fig. 5c), and $467 \mathrm{bp}$ fragments of the gK gene were also respectively amplified from the captured PRV (Fig. 5d). To investigate the viral load of $\operatorname{Im} 7$ Beads, $0.05 \mathrm{mg} \operatorname{Im} 7$ Beads immobilized with CL7CVN was applied to concentrate PRV from the $100-500 \mu \mathrm{l}$ samples $\left(10^{6}\right.$ viruses $\left./ \mu \mathrm{L}\right)$. When the virus sample volume increased from 100 to $300 \mu \mathrm{L}\left(10^{6}\right.$ viruses $(\mu \mathrm{L})$, the amount of the virus enriched increased to 3 times (Fig. 5e) and the enrichment efficiency of the virus also reached $89.64 \%$ (Fig. 5f).

\section{Discussion}

CVN has strong antiviral potential against a variety of enveloped viruses and may become a broad-spectrum antiviral drug (Smee et al. 2008; Buffa et al. 2009; Tiwari et al. 2009). However, the present production methods may be more or less defective. Here, we found the CL7-CVN we designed was highly soluble and functional expressed with excellent stability in the cytoplasm of E. coli (Vassylyeva et al. 2017). However, the mechanisms for increasing yield and improving solubility remain unclear. CL7 may affect the hydrophobicity and electrostatic repulsion of CVN analysis of $467 \mathrm{bp}$ fragments of gK gene of PRV captured by Im7 Beads with CL7-CVN. e Viral load analysis of Im7 Beads. f Changes in enrichment efficiency of PRV in increasing sample volume $\left(10^{6}\right.$ viruses $\left./ \mu \mathrm{L}\right)$

fusion protein, improving its solubility. Based on the thermotolerance and special structure design of CL7$\mathrm{CVN}$, we simplified the purification process of CVN, which greatly cut the production cost of this antiviral candidate. The expression and purification method described in this work allows for one-step purification of a wide range of traditionally antimicrobial peptide and heat-resistant protein.

Moreover, both purified CL7-CVN and CVN were biologically functional and had antiviral activities against PRV similar to others' enveloped viruses. Beside, we preliminarily performed the molecular docking of CVN and gD protein, indicating CVN and $\mathrm{gD}$ had the possibility of interaction but no details were described here. CVN contains two binding domains and inhibits HIV, influenza, Ebola, hepatitis $\mathrm{C}$, and herpes viruses by binding to viral envelope proteins via the high mannose glycans (Smee et al. 2008; Buffa et al. 2009; Tiwari et al. 2009). These viruses all have a large number of highly glycosylated envelope proteins. The S-protein of SARS-CoV-2 has at least 66 glycosylation sites, which may make it possible for CVN to be a potential antiviral drug. In the future, we will further explore the mechanism of action between CVN and enveloped viruses. 
Generally, efficiently concentrating virus from larger sample volumes can improve the detection signal. Based on the biological characteristics of CL7CVN, we explored a strategy for PRV enrichment from larger samples $(1 \mathrm{~mL})$ employing the affinity of CL7-CVN to Im7 Beads and PRV. This method also had some non-specific virus enrichment ( 20\%), which might be caused by the non-specific physical adsorption of Beads or $\operatorname{Im} 7$ Beads. However, the nonspecifically separated virus did not impact the overall assay as the qPCR assay for the virus detection was specific because of the specificity of the primers. Furthermore, the non-specific enrichment can be reduced by multiple washing prior to further downstream assays.

Compared to using magnetic nanoparticles, this strategy employing $\operatorname{Im} 7$ Beads can significantly cut the enrichment time via rapid separation. Generally, the smaller magnetic nanoparticles need to be separated overnight because of low magneto phoretic mobility. While the $\operatorname{Im} 7$ Beads can be separated by centrifugation in $1 \mathrm{~min}$. Additionally, the binding time between Im7 Beads, CL7-CVN and PRV can be optimized to decrease total enrichment time. Furthermore, the system utilized CL7-CVN for virus recognition, which did not depend on binding affinity of the antibody. Particularly, CVN could bind to the regions occluded by antibodies on glycoprotein. Compared to antibodies, CL7-CVN exhibits similar virus-binding ability with stable physicochemical property and more economical preparation methods (Wu et al. 2015).

In conclusion, we provided a simple, efficient and economical approach to produce the candidate antiviral drug CVN, and we explored a particular strategy to enrich whole virus for further downstream assays. Also, we wonder that the strategy may be extended to other targets and multiplexing. Future work will focus on rapid concentration and visual detection of viruses, as well as glycosylation detection of proteins.

Supplementary Information Supporting Information 1 Amino acid sequences for CL7-CVN: MGSKSNEPGKAT GEGKPVNNKWLNNAGKDLGSPVPDRIANKLRDKEFES FDDFRETFWEEVSKDPELSKQFSRNNNDRMKVGKAPKT RTQDVSGKRTSFELNHQKPIEQNGGVYDMDNISVVTPK RNIDIEGGGGGSGGGGSHHHHHHLEVLFQGPLGKFSQT CYNSAIQGSVLTSTCERTNGGYNTSSIDLNSVIENVDGSL KWQPSNFIETCRNTQLAGSSELAAECKTRAQQFVSTKIN LDDHMANMDGTLKYE

Supporting Information 2 SDS-PAGE analysis of purification of $\operatorname{Im} 7$ : Figure S1. pET28a-Im7 was successfully expressed and purified. The highly purified $\operatorname{Im} 7$ was then concentrated for protein immobilization onto the agarose beads to prepare $\operatorname{Im} 7$ Beads. The $\operatorname{Im} 7$ Beads were then used to capture viruses with CL7-CVN.wcl, whole cell lysates; sup, supernatant of cell lysates; pel, pellet of cell lysates; FT, flow through; EL, eluate.

Author contributions BW: Data curation (Supporting); Methodology (Lead); Writing-original draft (Lead). ZY: Data curation (Supporting); Methodology (Supporting); Validation (Supporting). DG: Data curation (Supporting); Methodology (Supporting); Validation (Supporting). FW: Visualization (Supporting). ML: Visualization (Supporting). GC: Visualization (Supporting). LM: Conceptualization (Supporting); Resources (Supporting); Writing-review \& editing (Supporting). XY: Conceptualization (Supporting); Resources (Lead); Writing-review \& editing (Lead).

Funding This work was supported by National Natural Science Foundation of China (Grant No. 31672561).

\section{Declarations}

Conflict of interest The authors declare that they have no financial or commercial conflict of interest.

\section{References}

Buffa V, Stieh D, Mamhood N, Hu Q, Fletcher P, Shattock RJ (2009) Cyanovirin-n potently inhibits human immunodeficiency virus type 1 infection in cellular and cervical explant models. J Gen Virol 90:234-243

Gao X, Chen W, Guo C, Qian C, Liu G, Ge F, Huang Y, Kitazato K, Wang Y, Xiong S (2010) Soluble cytoplasmic expression, rapid purification, and characterization of cyanovirin$\mathrm{N}$ as a His-SUMO fusion. Appl Microbiol Biotechnol 85:1051-1060

Kleiner-Grote GRM, Risse JM, Friehs K (2018) Secretion of recombinant proteins from E. coli. Eng Life Sci 18:532-550

Lusvarghi S, Lohith K, Morin-Leisk J, Ghirlando R, Hinshaw JE, Bewley CA (2016) Binding site geometry and subdomain valency control effects of neutralizing lectins on hiv1 viral particles. Acs Infectious Dis 2:882-891

Madeira LM, Szeto TH, Ma KC, Drake PMW (2016) Rhizosecretion improves the production of cyanovirin-n in nicotiana tabacum through simplified downstream processing. Biotechnol J 11(7):910-919

Mori T, Barrientos LG, Han Z, Gronenborn AM, Turpin JA, Boyd MR (2002) Functional homologs of cyanovirin-N amenable to mass production in prokaryotic and eukaryotic hosts. Protein Expr Purif 26:42-49

Sexton A, Drake PM, Mahmood N, Harman SJ, Shattock RJ, Ma JK (2006) Transgenic plant production of cyanovirin-N, an HIV microbicide. FASEB J 20:356-358 
Smee DF, Bailey KW, Wong MH, O"Keefe BR, Gustafson KR, Mishin VP, Gubareva LV (2008) Treatment of influenza a (h1n1) virus infections in mice and ferrets with cyanovirinn. Antiviral Res 80(3):266-271

Tiwari V, Shukla SY, Shukla D (2009) A sugar binding protein cyanovirin-n blocks herpes simplex virus type-1 entry and cell fusion. Antiviral Res 84(1):67-75

Vassylyeva MN, Klyuyev S, Vassylyev AD, Wesson H, Zhang Z, Renfrow MB, Wang H, Higgins NP, Chow LT, Vassylyev DG (2017) Efficient, ultra-high-affinity chromatography in a one-step purification of complex proteins. Proc Natl Acad Sci USA 114(26):e5138-e5147
Wu M, Zhang ZL, Chen G, Wen CY, Wu LL, Hu J, Xiong CC, Chen JJ, Pang DW (2015) Rapid and quantitative detection of avian influenza a(h7n9) virions in complex matrices based on combined magnetic capture and quantum dot labeling. Small 11(39):5280-5288

Wurm DJ, Slouka C, Bosilj T, Herwig C, Spadiut O (2017) How to trigger periplasmic release in recombinant escherichia coli: a comparative analysis. Eng Life Sci 17(2):215-222

Publisher's Note Springer Nature remains neutral with regard to jurisdictional claims in published maps and institutional affiliations. 